\title{
Electrical Impedance Studies of the Effect of a Buffer Layer on Organic Bulk Hetrojunction Solar Cells
}

\author{
C. K. Suman, Seunguk Noh, Seohee Kim, Sin-Doo LeE and Changhee LeE* \\ Department of Electrical Engineering and Computer Science, \\ Inter-university Semiconductor Research Center, Seoul National University, Seoul 151-742 \\ Donggu LeE and Jeongho PARK \\ Interdisciplinary Graduate Program in Nanoscience and Nanotechnology, \\ Inter-university Semiconductor Research Center Seoul National University, Seoul 151-742
}

(Received 13 August 2008)

\begin{abstract}
The effect of a buffer layer on organic bulk hetrojunction solar cells of poly(3-hexylthiophene) (P3HT) and [6,6]-phenyl-C61 butyric acid methyl ester fullerene derivative (PCBM) has been studied using impedance spectroscopy in the frequency range between $100 \mathrm{~Hz}$ and $1 \mathrm{MHz}$. The buffer layer interfaces with a metallic contact and with the organic bulk materials influence the efficiency and the stability of devices. Thermal annealing of the devices improves the interface with the buffer layer, thereby lowering the majority and the minority carrier injection voltage. The photocurrent density-voltage $(\mathrm{J}-\mathrm{V})$ characteristics show that the fill factor of the device with a molybdenum-oxide $\left(\mathrm{MoO}_{3}\right)$ buffer layer is higher than that of the device with a poly $(3,4$ ethylenedioxy-thiophene):polystyrenesulfonate (PEDOT:PSS) buffer layer. The equivalent circuit analysis for the PEDOT:PSS and the $\mathrm{MoO}_{3}$ buffer-layer devices shows that the device's capacitance and resistance for $\mathrm{MoO}_{3}$ is lower than that for PEDOT:PSS under illumination. The efficiency of the thermally-annealed PEDOT:PSS and $\mathrm{MoO}_{3}$ buffer-layer devices are 2.95 and $3.44 \%$, respectively. The lower efficiency of the PEDOT:PSS buffer-layer devices can be attributed to the formation of a thin insulator layer at the PEDOT:PSS/P3HT:PCBM interface under illumination.
\end{abstract}

PACS numbers: 72.40.+w, 85.60.Bt, 72.80.Le, 72.20.Jv, 73.40.Cg

Keywords: Organic solar cells, Bulk heterojunction, Buffer layer, Impedance spectroscopy, Interface, Metal diffusion, Traps

\section{INTRODUCTION}

The growing need for inexpensive renewable energy sources has attracted extensive studies of organic solar cells because of their advantages of large area, flexible shape, light weight, easy processing and low-cost production [1]. The structures of organic solar cells consist of donor-acceptor bilayer or bulk donor-acceptor hetrojunction networks sandwiched between a anode and a cathode [2-8]. In bulk hetrojunction (BHJ) solar cells consisting of conjugated polymers and fullerene derivatives, phase separation results in the formation of interpenetrating networks of polymers and fullerenes $[2,9]$. Under illumination, excitons form at the optically active materials and are dissociated at the interface of hetrojunction networks. The spatially separated holes and electrons move to the electrodes by hopping through interpenetrating networks of donor and acceptor materials [10]. Thus, the electrode and the morphology and the

\footnotetext{
*E-mail: chlee7@snu.ac.kr
}

potential difference of the organic semiconductor interface play an important role in determining the efficiency of a solar cell [11-14].

Bulk hetrojunction solar cells of P3HT and PCBM form Schottky contacts at the cathode due to exposure to air or moisture, leading to p-doping of P3HT [15]. The anode and the organic semiconductor contact are equally important for efficient devices. Recently, different types of buffer layers have been introduced to organic bulk hetrojunction solar cells [16]. The power conversion efficiency (PCE) of an ITO|P3HT:PCBM|Ca|Al solar cell without any buffer layer is $1.9 \%$ [11]. Different types of buffer layers for organic bulk hetrojunction solar cells, such as PEDOT:PSS (PCE $2.4 \%$ ), transition-metal oxides $\left(\mathrm{MoO}_{3}, \mathrm{~V}_{2} \mathrm{O}_{3}-\mathrm{PCE} \sim 3.33 \%\right)$ and p-type semiconducting nickel oxide ( $\mathrm{PCE} \sim 5.16 \%)$, have increased the power efficiency [13]. In addition, thermal annealing at high temperatures after device fabrication enhances the power efficiency [17-19]. However, despite the frequent use of buffer layers and thermal annealing for efficiency improvement in organic solar cells, the interfacial 


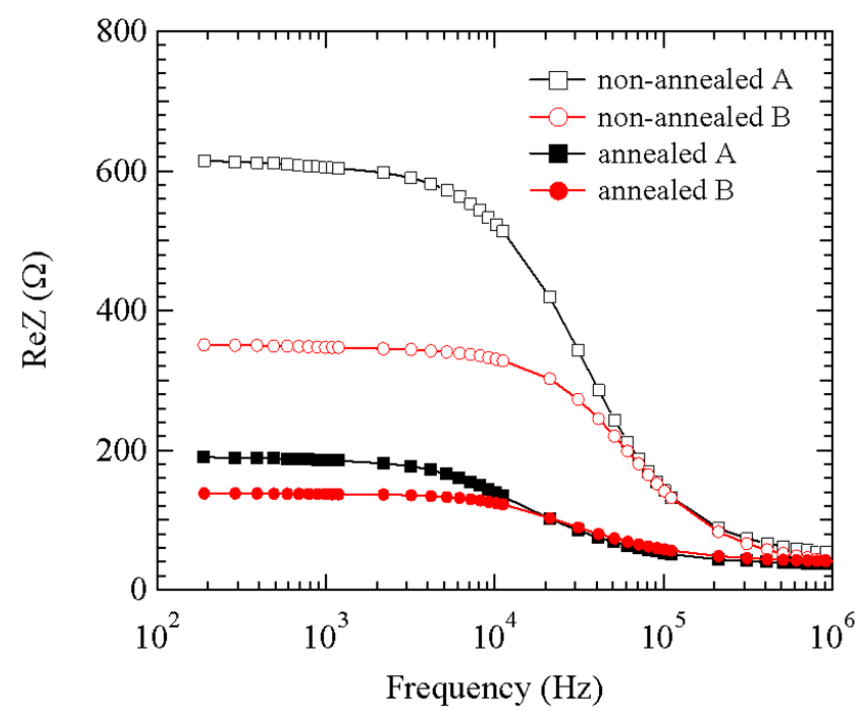

Fig. 1. Frequency-dependent real part of the impedance of annealed and non-annealed devices.

physical mechanisms of the buffer layer and the effect of thermal annealing are still not very clear.

Impedance spectroscopy is an important tool for studying the charge carrier dynamics, the interfacial characteristics, the dielectric properties of devices and equivalent circuit models [20]. In this investigation, we used electrical impedance spectroscopy to study the interfacial characteristics of the two different types of buffer layers used for improving the efficiency of organic bulk hetrojunction solar cell. The influence of thermal annealing on the buffer layer and the contacts was studied extensively.

\section{EXPERIMENT}

The indium-tin-oxide (ITO)-coated substrates were cleaned using isopropyl alcohol, de-ionized water, acetone and methanol in an ultrasonic bath and were dried in a vacuum oven prior to fabrication of the organic solar cells. To form the buffer layer between ITO and the active layer, a PEDOT:PSS (Baytron P) film was spincoated after an ultraviolet ozone (UVO) treatment and a $\mathrm{MoO}_{3}$ layer with a thickness of $5 \mathrm{~nm}$ was deposited by using thermal evaporation. These buffer layers were used in order to make ohmic contacts with the ITO anode. The optimized thicknesses of PEDOT:PSS and $\mathrm{MoO}_{3}$ were about $50 \mathrm{~nm}$ and $5 \mathrm{~nm}$, respectively [21]. A blend of P3HT and PCBM was spin-coated on the buffer layer; then, lithium fluoride $(\mathrm{LiF}) /$ Aluminum $(\mathrm{Al})$ were evaporated for top electrodes. The solar-cell area, which is the overlap area of the ITO and the $\mathrm{LiF} / \mathrm{Al}$ electrodes, is $0.16 \mathrm{~cm}^{2}$. The device structures are

Device A: ITO | PEDOT: PSS ( $\sim 50 \mathrm{~nm})$ P3HT:PCBM $(\sim 100 \mathrm{~nm})|\mathrm{LiF}| \mathrm{Al}$,

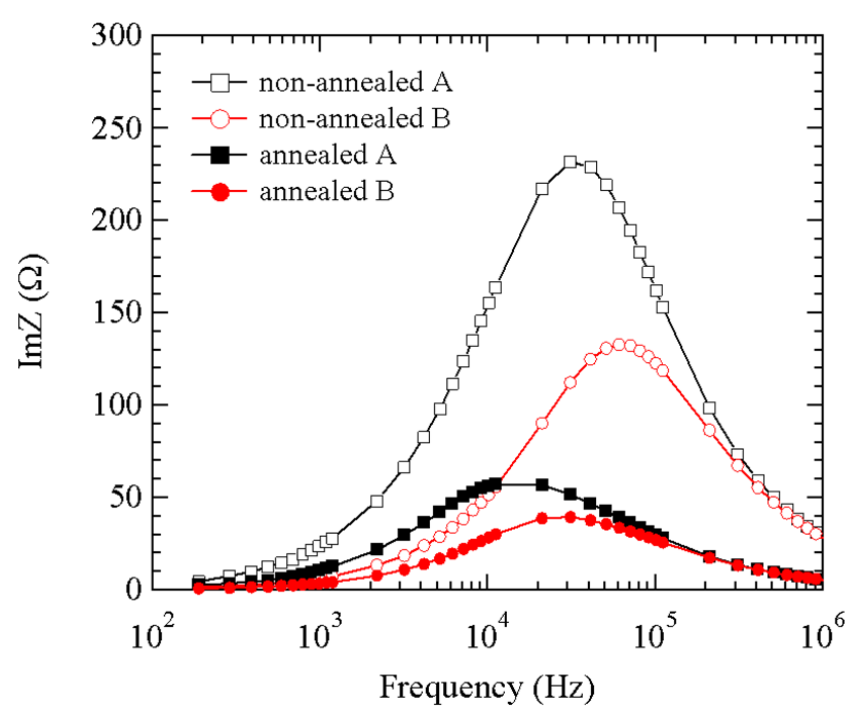

Fig. 2. Frequency-dependent imaginary part of the impedance of annealed and non-annealed devices.

\section{Device B: ITO $\left|\mathrm{MoO}_{3}(\sim 5 \mathrm{~nm})\right|$ P3HT:PCBM $(\sim 100 \mathrm{~nm})|\mathrm{LiF}| \mathrm{Al}$.}

After fabrication, the devices were thermally annealed in a dry nitrogen $\left(\mathrm{N}_{2}\right)$ glove box at $150{ }^{\circ} \mathrm{C}$ for 30 minutes. The photocurrent-voltage measurement was done at room temperature under an illumination of 100 $\mathrm{mW} / \mathrm{cm}^{2}$ (AM $1.5 \mathrm{G}$ ) by using a solar simulator (Newport $91160 \mathrm{~A})$. In order to prevent the perimeter effect under illumination, we covered the device except for an active cell area of $0.16 \mathrm{~cm}^{2}$, with a shadow mask. The impedance measurements were carried out in the broad frequency range by using an impedance analyzer (HP4192A). The oscillation amplitude of the AC voltage was maintained at $50 \mathrm{mV}$ for all measurements.

\section{RESULT AND DISCUSSION}

Figure 1 shows the real part of the complex impedance for different buffer layer devices (A and B) as functions of frequency without electrical biasing but under an illumination of $100 \mathrm{~mW} / \mathrm{cm}^{2}$. At low frequency, the value of the impedance for device $B$ is less than that of device A. After thermal annealing, the impedance value of $\mathrm{B}$ at low frequency is similarly lower than that of device A. The thermal annealing of the devices decreases the value of the impedance for both devices. This decrease in value may be attributed to improvements in the interface morphology between the buffer layer and the P3HT:PCBM blend film or anode and to an increase in the conductivity of the bulk P3HT:PCBM blend film.

Figure 2 shows the imaginary part of the impedance for non-annealed and annealed devices at zero bias and under illumination. The characteristic frequency $\omega_{c}$ (the frequency at which $\operatorname{ImZ}$ is maximum) is related 
Table 1. Device operation parameters and values of the elements of the modeled circuits.

\begin{tabular}{cccccc}
\hline \hline Devices & Efficiency $(\%)$ & $\mathrm{V}_{o c}(\mathrm{~V})$ & $\mathrm{R}_{s}(\Omega)$ & $\mathrm{R}_{p}(\mathrm{k} \Omega)$ & $\mathrm{C}_{p}(\mathrm{nF})$ \\
\hline Non-annealed A & 1.59 & 0.68 & 49.79 & 24.81 & 68.16 \\
Non-annealed B & 1.76 & 0.67 & 31.72 & 134.80 & 61.81 \\
Annealed A & 2.95 & 0.64 & 31.70 & 21.85 & 66.30 \\
Annealed B & 3.44 & 0.64 & 39.42 & 41.17 & 62.27 \\
\hline \hline
\end{tabular}

to the junction resistance of the devices in the complex impedance plane. This is a rough estimate of the junction properties of type A and B devices with and without annealing. The annealing of both devices shifts the characteristic frequency to lower frequency. The $\omega_{c}$ of device $\mathrm{B}$ for both the annealed and the non-annealed cases is higher than that of the corresponding cases of device A. These results indicate that the junction resistance is lower for a $\mathrm{MoO}_{3}$ buffer layer than for a PEDOT: PSS layer under illumination.

The complex impedance plane for devices can be presented in terms of the real and the imaginary parts of the impedance, $Z=Z^{\prime}+j Z^{\prime \prime}$, where $Z^{\prime}$ is the real part and $Z^{\prime \prime}$ is the imaginary part of the complex impedance. The imaginary impedance is plotted against real impedance for both annealed and non-annealed $\mathrm{A}$ and $\mathrm{B}$ devices under illumination in Figure 3(a). This Cole-Cole plot can be modeled as a combination of a resistance and capacitance network in series with a contact resistance [as shown in the inset of Figure 3(a)]. The modeled circuits can be used to determine the real and the imaginary parts of the impedance as

$$
\begin{aligned}
& Z=R_{s}+\frac{R_{P}}{1+\omega^{2} R_{P}^{2} C_{P}^{2}}+\frac{\omega R_{P}^{2} C_{P}}{1+\omega^{2} R_{P}^{2} C_{P}^{2}}, \\
& Z^{\prime}=R_{s}+\frac{R_{P}}{1+\omega^{2} R_{P}^{2} C_{P}^{2}} \text { and } \mathrm{Z}^{\prime \prime}=\frac{\omega \mathrm{R}_{\mathrm{P}}^{2} \mathrm{C}_{\mathrm{P}}}{1+\omega^{2} \mathrm{R}_{\mathrm{P}}^{2} \mathrm{C}_{\mathrm{P}}^{2}} .
\end{aligned}
$$

The estimated values of the capacitance and the resistance for the devices under illumination are given in Table 1. The series resistance (contact resistance) for device $\mathrm{B}$ is lower than that for device $\mathrm{A}$. The junction resistance and capacitance of device B is also lower than that of device $\mathrm{A}$, as explained in Figure 2.

Figure 3(b) shows the Cole-Cole plot of annealed devices in the dark, along with that of the non-annealed devices in the inset. The semicircle for the PEDOT:PSS buffer-layer device is smaller than that for the $\mathrm{MoO}_{3}$ buffer-layer device in the dark whereas the situation is reversed for both the annealed and the non-annealed cases under illumination. This is a clear indication of the interface effect of the buffer layer and the P3HT:PCBM blend film. The result indicates that the contact resistance is smaller in the dark, but becomes higher under illumination for the PEDOT:PSS buffer-layer device. This behavior can be interpreted as being due to the formation of a thin insulator layer at the PEDOT:PSS / P3HT:PCBM interface under illumination. The PEDOT:PSS thin film
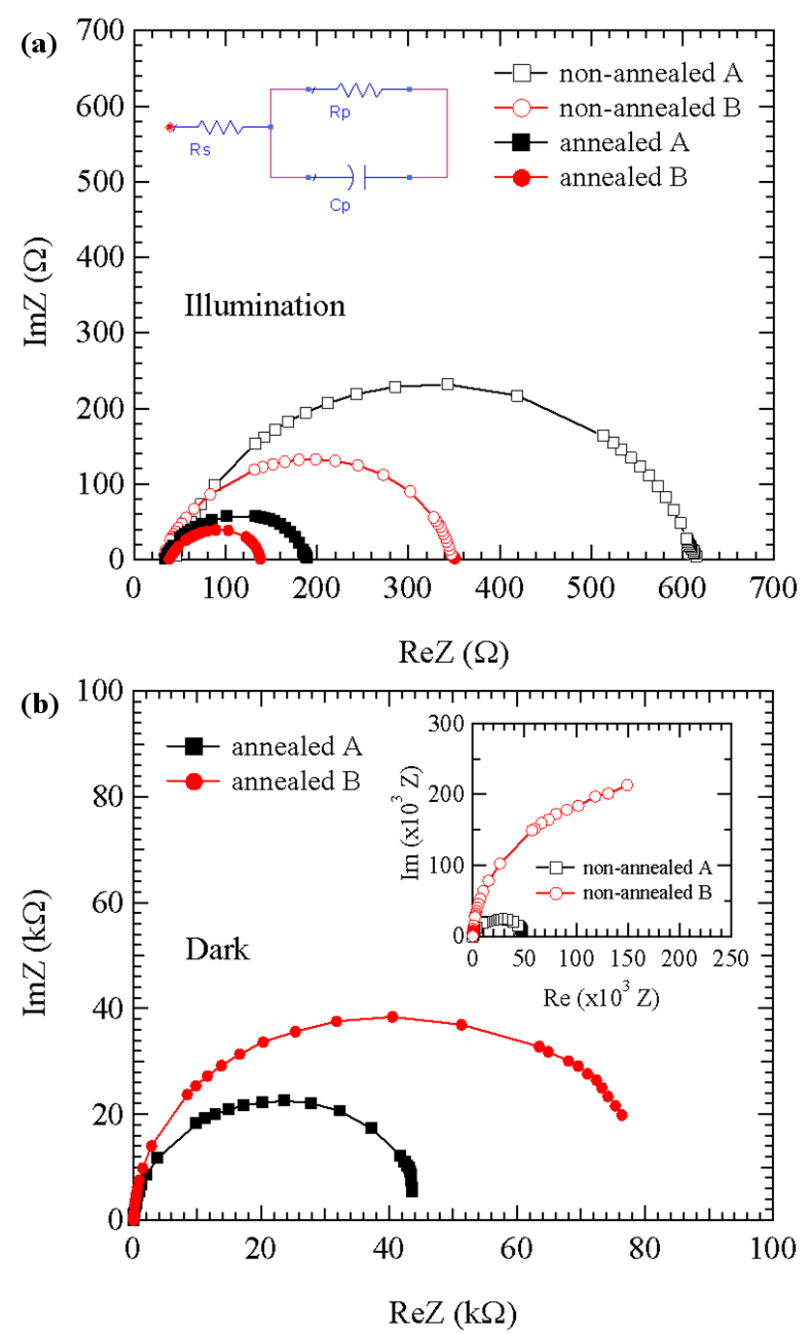

Fig. 3. Cole-Cole plot of annealed and non-annealed devices (a) under illumination (inset is the equivalent circuit) and (b) under dark (inset shows the non-annealed devices).

consists of p-doped PEDOT $\left(\mathrm{PEDOT}^{+}\right)$chain and PSS. The $\mathrm{PEDOT}^{+}$networks provide the conduction paths [22]. The electron injection into the PEDOT:PSS film can reduce $\mathrm{PEDOT}^{+}$to form neutral $\mathrm{PEDOT}^{0}$ (very low conducting state) $[22,23]$. Since the electrons photogenerated at the PEDOT:PSS / P3HT:PCBM interface can move into the PEDOT:PSS buffer layer, a thin undoped PEDOT layer (insulating $\mathrm{PEDOT}^{0}$ ) can be formed at the PEDOT:PSS / P3HT:PCBM interface under illumi- 


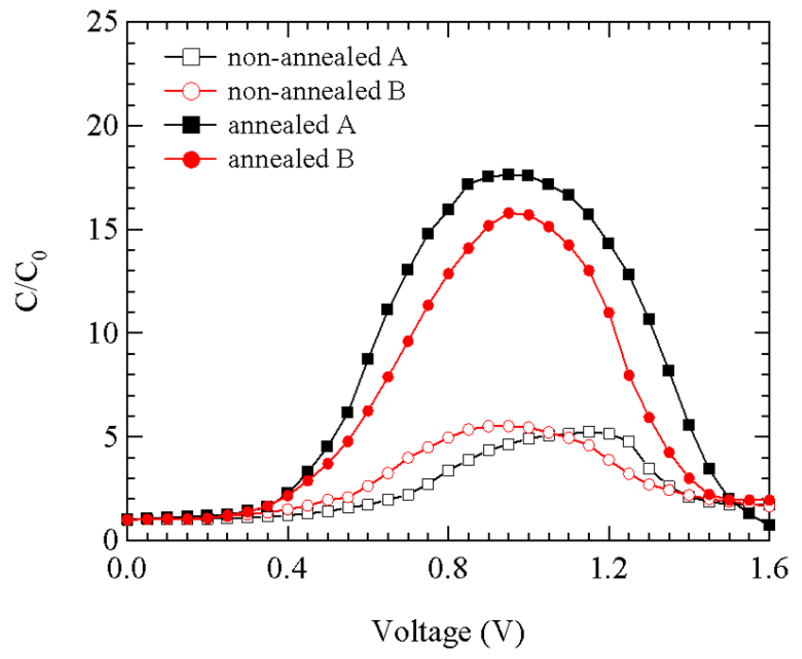

Fig. 4. Normalized capacitance $\left(\mathrm{C} / \mathrm{C}_{o}\right)$ for the devices as a function of the bias voltages at $100 \mathrm{~Hz}$, where $\mathrm{C}_{o}$ is the zero-voltage capacitance.

nation. This insulator layer increases the device's resistance, resulting in a change in the internal electric field inside the bulk P3HT:PCBM blend film. Both the stability and the efficiency are affected due to the insulator formation in the PEDOT:PSS buffer-layer devices.

Figure 4 shows the normalized capacitance $\left(\mathrm{C} / \mathrm{C}_{o}\right)$ for both devices, $\mathrm{A}$ and $\mathrm{B}$, at $100 \mathrm{~Hz}$ as functions of the bias voltage, where $\mathrm{C}_{o}$ is the capacitance of the devices at zero bias. The capacitances for both devices first increase with increasing bias voltage and after reaching maximum values, they start to decrease for further increases in the bias voltage. The increase in the value of the capacitance may be due to the charging of traps near the metallic contacts or the slow injection/extraction at the contact $[24,25]$. The decrease in capacitance may simply be attributed to significant electron and hole injections leading to neutralization of traps. Thermal annealing of the devices increases the capacitance to become around eighteen times the geometrical capacitance. The increase in the normalized capacitance after annealing suggests an increase in the number of metallic traps for both devices. The metallic traps that come from the diffusion of metal atoms to the organic materials have been the main cause for device degradation [26-28]. In the annealed devices, majority charge (hole) injection starts at lower voltages than it does for the non-annealed device and there is a small effect on the dual charge injection voltage. The annealing of devices enhances the conductivity of the bulk organic materials and improves the interfaces of the anode/cathode with the organic materials. There is small effect of annealing on the cathode side become the minority injection voltage decreases approximately from $1.2 \mathrm{~V}$ to $0.9 \mathrm{~V}$.

Figure 5 shows the current density-voltage plot for non-annealed devices [Figure 5(a)] and annealed devices [Figure 5(b)] in dark and under an illumination of 100
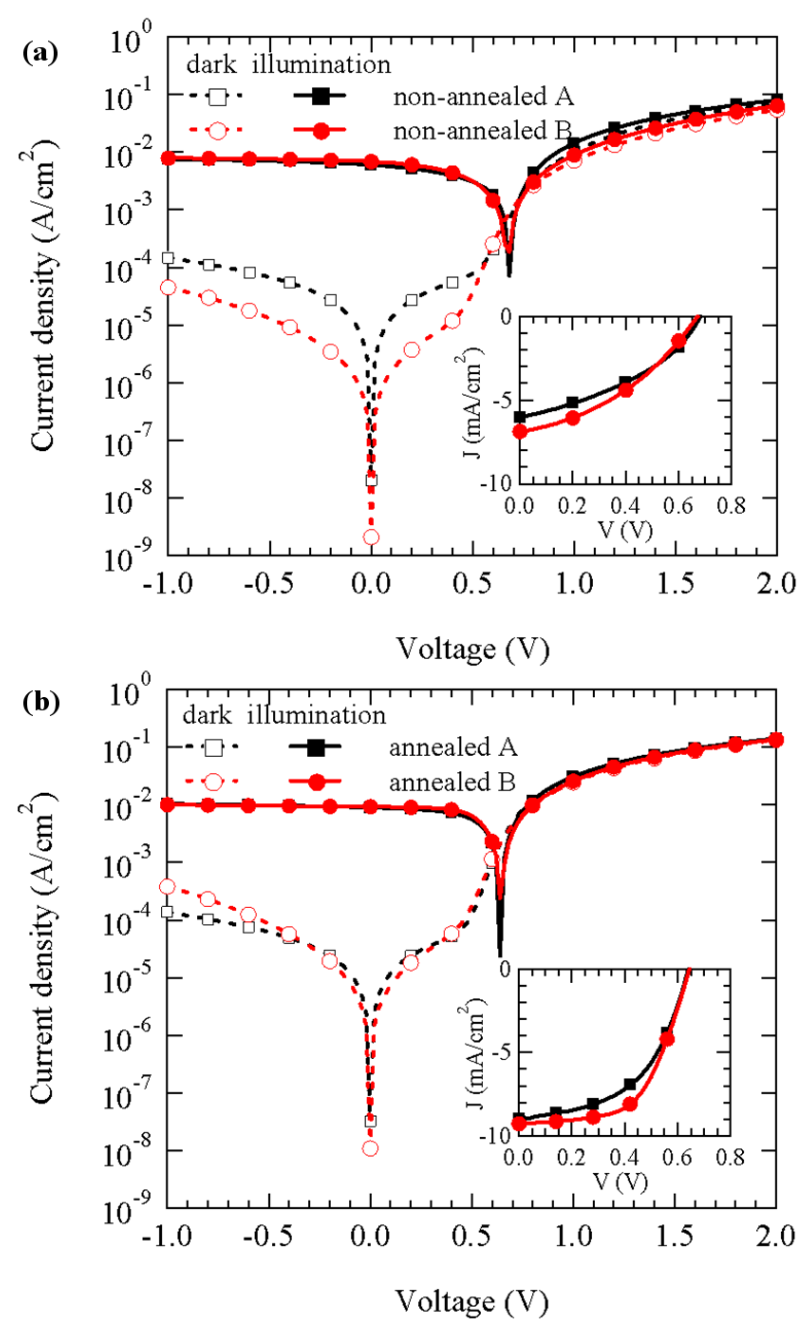

Fig. 5. Current density-voltage characteristics under dark and illumination for (a) non-annealed and (b) annealed devices (insets of both figures are the photovoltaic responses).

$\mathrm{mW} / \mathrm{cm}^{2}$. The insets of both figures show the photovoltaic effects of the devices. For both the PEDOT:PSS and the $\mathrm{MoO}_{3}$ buffer layers, the short-circuit current $\left(\mathrm{I}_{s c}\right)$, the fill factor $(\mathrm{FF})$ and the efficiency are increased after thermal annealing while the open-circuit voltage $\left(\mathrm{V}_{o c}\right)$ is slightly decreases, as reported previously [17]. The rectification is influenced by both the buffer layer and the annealing process. The device performance with the $\mathrm{MoO}_{3}$ buffer layer is better than that with PEDOT:PSS. In particular, compared with the $\mathrm{MoO}_{3}$ buffer-layer devices, the PEDOT:PSS buffer-layer devices show smaller $\mathrm{FF}$ while $\mathrm{V}_{o c}$ is the same [see Figure 5(b)]. Since a large serial resistance and a small parallel resistance (shunt) tend to decrease the fill factor [29], this result again corroborates that the PEDOT:PSS buffer layer has a higher serial resistance under illumination than the $\mathrm{MoO}_{3}$ buffer layer. In addition, the larger FF and $\mathrm{I}_{s c}$ of the $\mathrm{MoO}_{3}$ buffer layer devices imply that the $\mathrm{MoO}_{3}$ buffer layer can act as an exciton blocking 
layer so that excitons can be dissociated more efficiently in the bulk P3HT:PCBM blend film.

\section{CONCLUSION}

We have concluded that the interface of buffer layer plays an important role in organic bulk hetrojunction solar cells. The bulk resistance of device A (PEDOT:PSS buffer layer) increases under illumination in comparison to device $\mathrm{B}\left(\mathrm{MoO}_{3}\right.$ buffer layer $)$. This may be due to the formation of a thin insulator layer at the PEDOT:PSS interface due to reactions with electrons. The efficiency of the solar cell is limited by the interface insulator. Thermal annealing of the devices increases the conductivity of the bulk organic materials and improves the cathode interface. After both devices had been annealed, the minority carrier injection voltages decreased and the number of metallic interface traps increased. The $\mathrm{MoO}_{3}$ buffer-layer solar cell (Efficiency $\sim 3.44 \%$ ) gives better performance than the PEDOT: PSS buffer-layer device (Efficiency $\sim 2.95 \%$ ).

\section{ACKNOWLEDGMENTS}

This work was supported by a Korea Science and Engineering Foundation (KOSEF) NRL Program grant (R0A-2008-000-20108-0) funded by the Korea government (MEST).

\section{REFERENCES}

[1] Organic Photovoltaics: Concepts and Realization, Vol. 60, edited by C. J. Brabec, V. Dyakonov, J. Parisi and N. S. Sariciftci, (Springer, Berlin, Germany, 2003).

[2] G. Yu, J. Gao, J. C. Hummelen, F. Wudl and A. J. Heeger, Science 270, 1789 (1995).

[3] J. Y. Park, S. B. Lee, Y. S. Park, Y. W. Park, C. H. Lee, J. I. Lee and H. K. Shim, Appl. Phys. Lett. 72, 2871 (1998).

[4] R. Koeppe, N. S. Sariciftci, P. A. Troshin and R. N. Lyubovskaya, Appl. Phys. Lett. 87, 244102 (2005).

[5] S. Alem, R. de Bettignies, J.-M. Nunzi and M. Cariou, Appl. Phys. Lett. 84, 2178 (2004).

[6] S. C. Jain, T. Aernout, A. K. Kapoor, V. Kumar, W. Geens, J. Poortmans and R. Mertens, Synth. Met. 148,
245 (2005).

[7] S. E. Shaheen, C. J. Brabec and N. S. Sariciftci, Appl. Phys. Lett. 78, 841 (2001).

[8] Y.-J. Ahn, G.-W. Kang, C. H. Lee, I. S. Yeom and S. H. Jin, Synth. Met. 137, 1447 (2003).

[9] C. H. Lee, G. Yu, D. Moses, K. Pakbaz, C. Zhang, N. S. Sariciftci, A. J. Heeger and F. Wudl, Phys. Rev. B 48, 15425 (1993).

[10] M. Glatthaar, N. Mingirulli, B. Zimmermann, T. Ziegler, R. Kern, M. Niggemann, A. Hinsch and A. Gombert, Phys. Stat. Sol. (a) 202, R125 (2005).

[11] V. Shrotriya, G. Li, Y. Yao, C. W. Chu and Y. Yang, Appl. Phys. Lett. 88, 508 (2006).

[12] F. L. Zhang, A. Gadisa, O. Inganas, M. Svensson and M. R. Andersson, Appl. Phys. Lett. 84, 3906 (2004).

[13] M. D. Irwin, D. B. Buchholz, A. W. Hains, R. P. H. Chang and T. J. Marks, Proc. Nat. Acad. Sci. 105, 2783 (2008).

[14] M. M. Erwin, J. McBride, A. V. Kadavanich and S. J. Rosenthal, Thin Solid Films 409, 198 (2002).

[15] Z. Tan, C. Yang, E. Zhou, X. Wang and Y. Li, Appl. Phys. Lett. 91, 023509 (2007).

[16] I. S. Yoo, M. J. Lee, C. H. Lee, D. W. Kim, I. S. Moon and D. H. Hwang, Synth. Met. 153, 97 (2005).

[17] F. Padinger, R. S. Rittberger and N. S. Sariciftci, Adv. Funct. Mater. 13, 85 (2003).

[18] H. J. Kim, W.-W. So and S.-J. Moon, J. Korean Phys. Soc. 48, 441 (2006).

[19] Y. H. Lee, I. H. Oh, K. W. Lee, S. M. Yoon and C. E. Lee, J. Korean Phys. Soc. 51, 1457 (2007).

[20] S. H. Kim, K. H. Choi, H. M. Lee, D. H. Hwang, L. M. Do, H. Y. Chu and T. Zyung, J. Appl. Phys. 87, 882 (2002).

[21] J.-Y. Yun, J.-J. Yang, Y.-T. Hong, C. H. Lee, W. J. Song and Y. J. Sung, J. Korean Phys. Soc. 53, 1660 (2008).

[22] H.-J. Ha and O.-H. Kim, Appl. Phys. Lett. 93, 033309 (2008).

[23] J. S. Kim, R. H. Friend, I. Grizzi and J. H. Burroughes, Appl. Phys. Lett. 87, 023506 (2005).

[24] G. Garcia-Belmonte, A. Munar, E. M. Barea, J. Bisquert, I. Ugarte and R. Pacios, Org. Elect. 10, 1016 (2008).

[25] V. Shrotriya and Y. Yang, J. Appl. Phys. 97, 054504 (2005).

[26] E. I. Haskal, A. Curioni, P. F. Seidler and W. Andreoni, Appl. Phys. Lett. 71, 1151 (1997).

[27] S. T. Lee, Z. Q. Gao and L. S. Hung, Appl. Phys. Lett. 75, 1404 (1999)

[28] A. R. Schlatmann, D. Wilms Floet, A. Hilberer, F. Garten, P. J. M. Smulders, T. M. Klapwijk and G. Hadziioannou, Appl. Phys. Lett. 69, 1764 (1996).

[29] C. J. Brabec, N. S. Sariciftci and J. C. Hummlen, Adv. Func. Mater. 11, 15 (2001) 